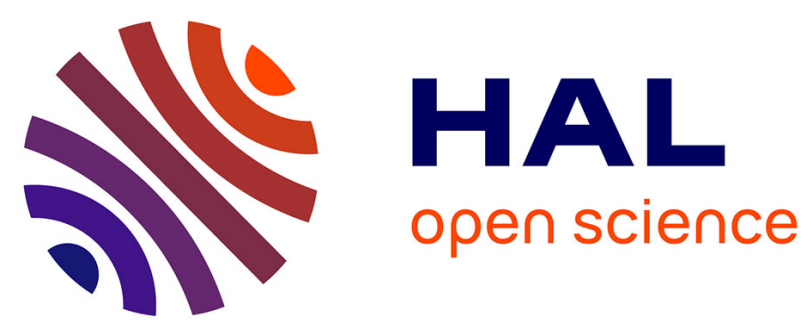

\title{
Loss assessment in random crystal polarity gallium phosphide microdisks grown on silicon
}

Rasool Saleem-Urothodi, Julie Le Pouliquen, Tony Rohel, Rozenn Bernard, Christelle Pareige, Alejandro Lorenzo-Ruiz, Alexandre Beck, Antoine Létoublon, Olivier de Sagazan, Charles Cornet, et al.

\section{To cite this version:}

Rasool Saleem-Urothodi, Julie Le Pouliquen, Tony Rohel, Rozenn Bernard, Christelle Pareige, et al.. Loss assessment in random crystal polarity gallium phosphide microdisks grown on silicon. Optics Letters, 2020, 45 (16), pp.4646. 10.1364/OL.399935 . hal-02926153

\section{HAL Id: hal-02926153 https://hal.science/hal-02926153}

Submitted on 31 Aug 2020

HAL is a multi-disciplinary open access archive for the deposit and dissemination of scientific research documents, whether they are published or not. The documents may come from teaching and research institutions in France or abroad, or from public or private research centers.
L'archive ouverte pluridisciplinaire HAL, est destinée au dépôt et à la diffusion de documents scientifiques de niveau recherche, publiés ou non, émanant des établissements d'enseignement et de recherche français ou étrangers, des laboratoires publics ou privés. 


\title{
Loss assessment in random crystal polarity gallium phosphide microdisks grown on silicon
}

\author{
Rasool Saleem-Urothodi, ${ }^{1}$ Julie Le Pouliquen, ${ }^{1}$ Tony Rohel, ${ }^{1}$ Rozenn \\ Bernard, ${ }^{1}$ Christelle Pareige, ${ }^{1}$ Alejandro lorenzo-Ruiz, ${ }^{1}$ Alexandre \\ Beck, ${ }^{1}$ Antoine létoublon, ${ }^{1}$ Olivier De Sagazan, ${ }^{2}$ Charles Cornet, ${ }^{1}$ \\ YANNICK DUMEIGE, ${ }^{1}$ YOAN LÉGER ${ }^{1,}{ }^{*}$
}

${ }^{1}$ Univ Rennes, INSA de Rennes, CNRS, Institut FOTON - UMR6082, F-35000 Rennes, France

${ }^{2}$ Institut d'Électronique et des Télécommunications de Rennes, UMR CNRS 6164, F-35042 Rennes, France

*Corresponding author: yoan.leger@insa-rennes.fr

\begin{abstract}
III-V semiconductors grown on silicon recently appeared as a promising platform to decrease the costs of photonic components and circuits. For nonlinear optics, specific features of the III-V crystal arising from the growth on the nonpolar Si substrate and called antiphase domains, offer a unique way to engineer the second order properties of the semiconductor compound. Here, we demonstrate the fabrication of microdisk resonators at the interface between a gallium phosphide layer and its silicon substrate. The analysis of the whispering gallery mode quality factors in the devices allows the quantitative assessment of losses induced by a controlled distribution of antiphase domains in the GaP layer and demonstrate the relevance of such a platform for the development of polarity-engineered III-V nonlinear photonic devices on silicon.
\end{abstract}

III-V semiconductor photonics has for long obtained the monopole on integrated light emitter technologies.[1,2] Significant advances are now reported in other domains of integrated photonics such as nonlinear devices.[3-5] Despite the promising nonlinear optical properties of these materials, coupled to band gap engineering, heterostructure fabrication and advanced technological processing, III-V semiconductors should compete with other platforms such as lithium niobate which also benefited from recent advances on photonic integration.[6,7] Lithium niobate is also sometimes presented as a cheaper solution compared to III$\mathrm{V}$, which require expensive epitaxy costs, lying mainly in substrate costs. This argument may not hold in the future with the emergence of III-V photonic devices directly grown on silicon substrates.[8] III$\mathrm{V}$ lasers on silicon now feature performances as good as their equivalent on III-V substrates.[9]

In this work, we investigate the potential of III-V semiconductors on silicon for nonlinear photonics. We focus in particular on an asset that arises from the growth of the III-V polar material on non-polar
Si. During the growth, the crystal polarity of the III-V material can be locally reversed due to the formation of antiphase domains (APDs).[10-12] While the APD distribution is generally random, some groups intensively work on its tailoring, from the annihilation of minority domains[13] to the periodic-poling of the crystal,[10] which has proven to be so efficient for second order nonlinear optics. The randomicity of the APD distribution could also be used to relax phase matching in $2^{\text {nd }}$ order processes and provide tunability to devices $[14,15]$ or even to suppress $2^{\text {nd }}$ order processes at the benefit of $\chi^{(3)}$ nonlinearity.[15] However, the antiphase boundaries (APBs) are also often considered as defects that could impact optical propagation[16] and an experimental assessment of the induced losses remains, to our knowledge, unreported.

Here, we demonstrate the fabrication of gallium-phosphide microdisks on silicon and investigate the contributions to optical losses in these devices. The resonators are fabricated from a GaP layer directly grown on a silicon substrate, and featuring a random crystal polarity distribution with controlled parameters. The statistical investigation of the whispering gallery modes (WGM) for a set of disks of different radii coupled to a detailed characterization of the APD distribution and sidewall roughness allows us to identify the different contributions to optical losses in these devices and assess quantitatively an upper limit of $10 \mathrm{~dB} / \mathrm{cm}$ for the losses induced by APDs in the GaP layer.

The $400 \mathrm{~nm}$ thick GaP epilayer was grown on a vicinal $6^{\circ}$-off (001) silicon substrate. After chemical preparation of the substrate using $\mathrm{HF}-1 \% / \mathrm{UV}-\mathrm{O}_{3} / \mathrm{HF}-1 \%[17]$ and rapid rise at $800^{\circ} \mathrm{C}$ for hydrogen desorption, $10 \mathrm{~nm}$ of GaP were first grown using migration enhanced epitaxy at $350^{\circ} \mathrm{C}$. The substrate temperature was then progressively increased from 580 to $660^{\circ} \mathrm{C}$ to grow the rest of the GaP layer. A V/III ratio slightly above 1 was used. The III$\mathrm{V}$ layer also includes three 2-nm thick $\mathrm{Al}_{0.2} \mathrm{GaP}$ wells positioned at $10 \mathrm{~nm}, 35 \mathrm{~nm}$ and $50 \mathrm{~nm}$ from the Si interface. Their role in the annihilation of minority domains has been reported in [18]. Here 
we couple this method to the fine tuning of V/III ratio and growth temperature on a vicinal substrate [19] to tailor the spatial distribution of APDs and obtain domains size in the $100 \mathrm{~nm}$ range.

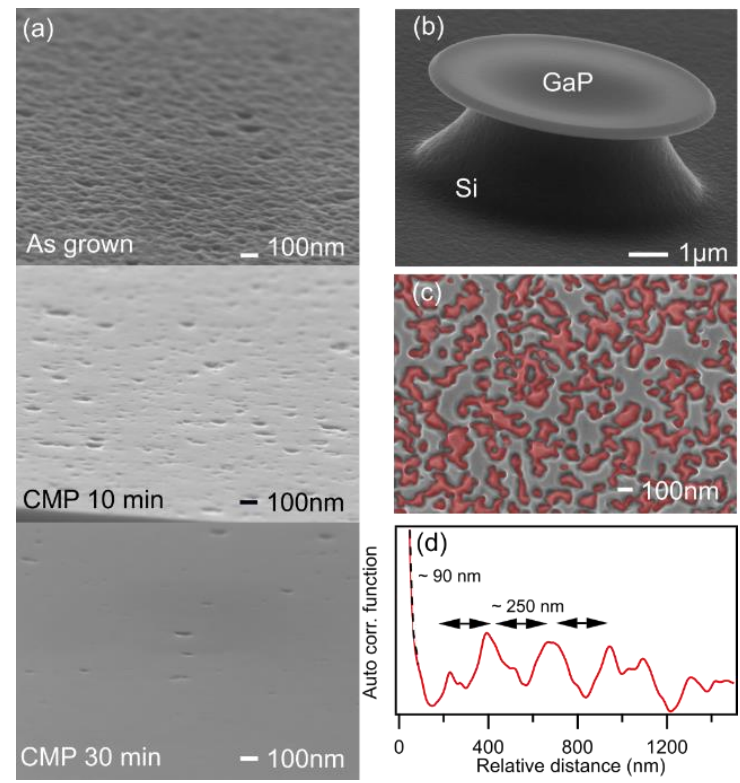

Fig. 1. (a) Scanning electron microscope (SEM) images of the surface of a GaP film grown on $\mathrm{Si}$, planarized with $\mathrm{H}_{3} \mathrm{PO}_{4} \mathrm{CMP}$ (from top to bottom: as grown, after $10 \mathrm{~min}$ and after $30 \mathrm{~min}$ of CMP. (b) SEM image of a $\mathrm{GaP} /$ Si microdisk processed with the sample in a) after 1h CMP.(c) SEM image of the antiphase domain distribution of the GaP/Si film. (d) Autocorrelation function calculated from the APD distribution.

In its virgin state, the sample shows a surface roughness of $8.4 \mathrm{~nm}$ RMS, typical of III-V/Si epilayers with emerging APDs,[20] which is not suitable for the realization of photonic components. Chemical mechanical polishing (CMP) using phosphoric acid at $1 \%$ was thus used to reduce the GaP surface roughness as presented in Fig.1 a). After 30 min of CMP, a removal of $100 \mathrm{~nm}$ of GaP already ensured a flat surface with low concentration of surface defects. Polishing was further performed to decrease the layer thickness to $250 \mathrm{~nm}$ and obtain a roughness of $1 \mathrm{~nm}$ RMS. The sample was then cut into pieces to perform both technological processing of the microdisk and further analysis of the APD distribution.

Even though emerging APDs strongly impact the roughness of $\mathrm{GaP} / \mathrm{Si}$ epilayers, a direct correlation of the sample topography to the crystal polarity distribution remains speculative. On the contrary, after CMP, the sample can be quickly dip into GaP Etch commercial solution to reveal APBs. Scanning electron microscopy (SEM) and atomic force microscopy (AFM) were then employed to identify domains of opposite polarity and statistically analyze the crystal polarity distribution. The SEM image analysis for this sample is shown in Fig. 1 c). On the SEM image (upper panel), one clearly observes the network of valleys resulting from the fast etching of APBs on the polished surface, where minority domains are highlighted in red. A binarized image was processed from this identification and used to compute the autocorrelation function presented in the lower panel of Fig.1c) and d). In such a binary distribution, the exponential decay of the central peak is directly related to the size of the minority domains and the distance between satellite peaks reflects the mean distance between homophase domains. Here, the average size for minority domains is $90 \pm 5 \mathrm{~nm}$ and the mean distance between them is $250 \pm 60 \mathrm{~nm}$. From integration of the binarized surface, the average phase in the sample was measured to be 0.3 (that is to say, $65 \%$ of the surface occupied by +1 domains and $35 \%$ by -1 domains).

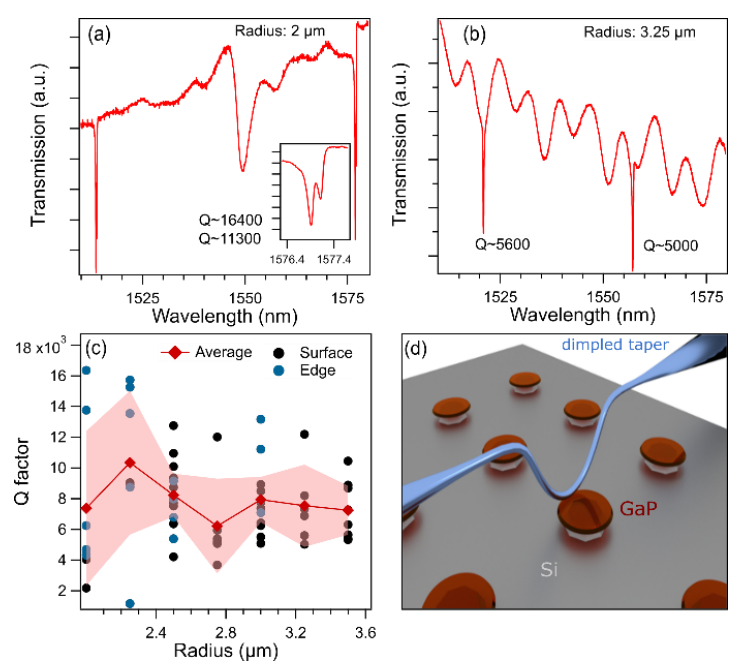

Fig.2 (a) and (b) Typical transmission spectra of microdisks of different radius. The inset in a) is a close-up view of the doublet structure caused by back-scattering. (c) Statistical distribution of measured Q factors of TE WGMs vs disk radius. The coupling method is indicated with black (surface-coupling)) and blue (edge-coupling) markers (in red: averaged $\mathrm{Q}$ factor value and $25-75 \%$ confidence interval). (d) Sketch of the dimpled tapered fiber coupling apparatus.

Another piece of sample was used to fabricate suspended GaP microdisks as shown in Fig.1 b). The GaP/Si sample was masked with $\mathrm{SiN}_{\mathrm{x}}$, doped $\mu$-Si and PMMA. We performed e-beam lithography and a Ni lift-off to define a mask to etch the $\mu$-Si/SiN ${ }_{x}$ stack with $\mathrm{CF}_{4}$ ICP-RIE. The Ni mask was removed with $\mathrm{HNO}_{3}$ and the pattern was then transferred to the $\mathrm{GaP}$ layer using $\mathrm{Cl}_{2} / \mathrm{Ar}$ ICPRIE. After mask removal with $\mathrm{CF}_{4}$ ICP-RIE, microdisks were partially released by underetching the $\mathrm{Si}$ with isotropic $\mathrm{SF}_{6} \mathrm{RIE}$ which also bevels slightly the GaP rims.[21] The sample features a 2D-array of microdisks with increasing size. Their radii vary from 2 $\mu \mathrm{m}$ to $3.5 \mu \mathrm{m}$. The sample was then placed under an optical microscope featuring nano-positioning stages for the sample and for the optical coupling apparatus. The latter consists in a single mode optical fiber tapered to a diameter of $1 \mu \mathrm{m}$ and dimpled to couple individual microdisks of the array as illustrated in Fig.2 d).[22] For each disk, several coupling positions of the fiber (on the edge of the disk and onto the surface either close to the edge or to the center) were tested to find under-coupling conditions. The spectrally-resolved optical transmission through the fiber was obtained by use of an Agilent 8164A Lightwave Measurement System with $8 \mathrm{pm}$ spectral resolution. In the following, we consider that this technique provides a good estimation of the intrinsic $Q$ factors of the disks $Q_{i}=\left(1 / Q_{\text {rad }}+1 / Q_{R}+1 / Q_{A P D}\right)^{-1}$, which can be altered by radiation losses $\left(Q_{\text {rad }}\right)$, scattering losses on sidewall roughness $\left(Q_{R}\right)$ and volumic losses due to absorption in antiphase boundaries $\left(Q_{A P D}\right)$. We neglect contributions from other structural defects. Thanks to the CMP process, we can neglect the contribution of surface losses. 
Typical transmission spectra for two disks of different radii are presented in Fig. 2a) and b). In the wavelength span of the laser, a small number of WGMs are observed. With a thickness of $250 \mathrm{~nm}$, the GaP layer is monomode for both TE and TM modes. The calculation of the free spectral range of WGMs with radial order $n=1$ reveals that we mostly observe two sets of WGM which we identify as $n=1$ TE and TM modes. Due to the small thickness of our disks, the effective refractive index is small for TM modes, limiting $Q_{\text {rad }}$ of these modes to a few $10^{2}$ as observed in Fig.2 a). The larger effective index of TE modes allows for higher intrinsic $Q$ factors as observed on both spectra. Theoretically, one could expect values of $Q_{\text {rad }}$ in the $10^{6}$ range even for $2 \mu \mathrm{m}$ radius disks. We will restrict the loss analysis to the $\mathrm{Q}$ factors of TE modes in the following. The absence of other sets of TE modes with higher radial order is attributed to the lack of optical confinement between the GaP disk and the $\mathrm{Si}$ pedestal (the undercutting distance is no larger than $1 \mu \mathrm{m}$ ). In most cases, the measured TE modes feature a mode splitting typical of back-scattering coupling in agreement with the measured roughness.[23,24]

Figure 2 c) shows the $Q$ factor distribution for 59 TE $n=1$ WGMs measured on 37 microdisks of various radii. The $Q$ factors vary from around 2500 to 16000 . The averaged $Q$ factor, $\bar{Q}$, vs radius is also plotted with red markers and shading is used to represent the 25$75 \%$ confidence interval. We observe no significant variation of $\bar{Q}$, as a function of radius, indicating that all $Q$ factors are limited by some loss contribution. The averaged value is close to the $\mathrm{Q}$ factors obtained in previous works on $\mathrm{GaP} / \mathrm{AlGaP} / \mathrm{GaP}$ microdisks fabricated using a similar technological process.[21] On Fig.2 c), one observes that the confidence interval gets narrower with increasing radius. This will be discussed in the following.

One important source for optical loss is sidewall roughness. In our samples, this contribution can be strong due to the lift-off process used to define the disks, known to induce more roughness than metal-free masking.[25] To assess it, we extracted from SEM images the lateral microdisk roughness versus perimeter position $x_{p}=R \varphi$ as shown in Fig.3 a) and c) (inset). The measured roughness is here limited by the $5 \mathrm{~nm}$ resolution of the SEM. The microdisk profiles were then both uploaded in a finite element method (FEM) environment to get a direct estimation of $Q_{R}$ on individual roughness distribution (Fig.3 b) and statistically studied to get a deeper insight on the roughness impact (Fig.3 c). On the specific example of Fig.3 a) and b), sidewall roughness induces significant scattering of the optical power outside and within the disk. In this simulation, the $Q$ factor of the WGM at $1.57 \mu \mathrm{m}$ is found to be 30000 . This value does not account for the absence of the optical confinement in the central part of the disk where it is supported by the Si pedestal.

The statistical analysis of the sidewall roughness allows us to highlight the origin of this strong loss contribution. The autocorrelation function of the sidewall roughness is plotted in Fig.3 c). It shows a short scale component, revealed in the fast decaying central peak of the autocorrelation and a long scale one appearing in the form of satellite peaks. The short scale component shows the exponential decay typical of random roughness with correlation length $S_{c, \text { short }}=70 \pm 5 \mathrm{~nm}$. Conversely, the long scale component is pseudo-periodical with a correlation length (or pseudo-period) $S_{c, \text { long }}=650 \pm 50 \mathrm{~nm}$. These roughness components have individual RMS values $\sigma_{\text {short }}=\sigma_{\text {long }}=5 \mathrm{~nm}$ so that the combined roughness has been measured to $7 \mathrm{~nm}$ RMS.
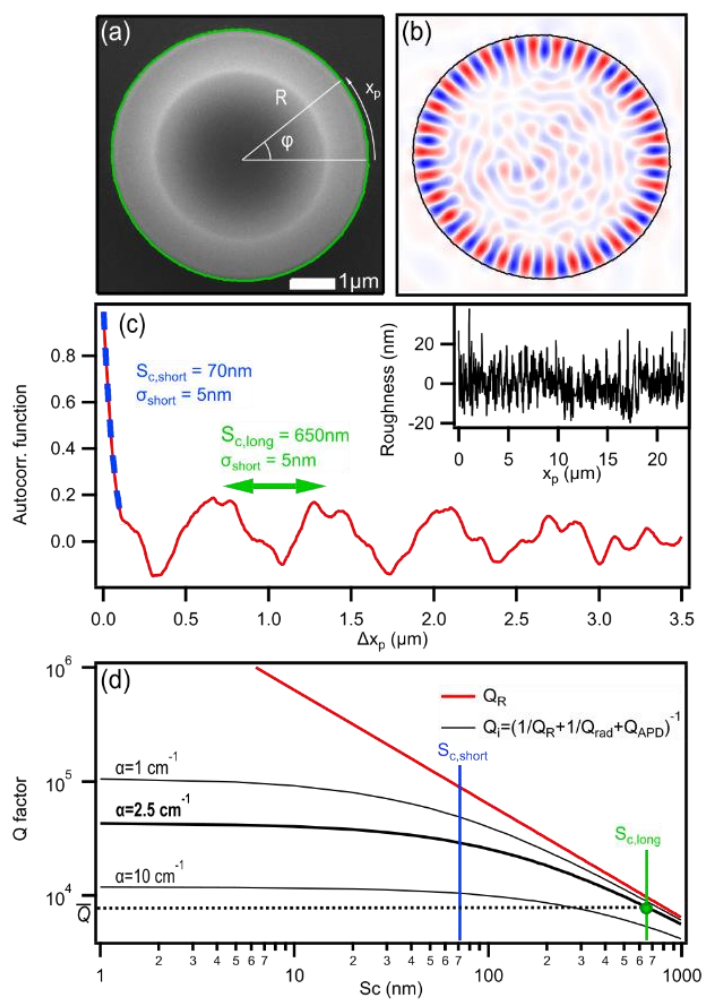

Fig. 3. (a) SEM view of a GaP/Si microdisk used to extract the lateral profile of the disks. (b) FEM simulation of a TE WGM at $1.57 \mu \mathrm{m}$ using this profile. (c) Autocorrelation function of the lateral roughness profile (given in inset). (d) Theoretical dependence of the roughness induced $Q$ factor $Q_{R}$ vs roughness correlation length $\mathrm{Sc}$ (red) as well as the intrinsic $\mathrm{Q}$ factor of the disk $Q_{i}$ for different APD-induced volumic losses (black plots) and matching point with the experimental average $Q$ factor $\bar{Q}$. The curve is plotted for $\sigma=5 \mathrm{~nm}$ and $2 \mu$ m radius disk.

The impact of sidewall roughness has been previously studied using both many-scatterers approach[23] and volume current method for distributed roughness.[24,26] Following the analysis proposed in Ref.[26] one can plot the roughness-induced contribution $Q_{R}$, as a function of the correlation length $S_{c}$ for the TE modes of a thin microdisk, as presented in Fig. $3 \mathrm{~d}$ ). The calculation is performed for a fixed RMS value of $5 \mathrm{~nm}$. The plot shows that the only effect of the measured short scale component should lead to $Q$ factors around $10^{5}$. On the contrary, long scale components of roughness are expected to affect more strongly the $\mathrm{Q}$ factor with average value around $10^{4}$. This said, the contribution we observe is not random but pseudo-periodical. As a consequence, it is not expected to affect equivalently all WGMs, depending on their azimuthal order. Moreover, the pseudo-period is only one order of magnitude smaller than the perimeter of the disks under scrutiny. From disk to disk, the roughness distribution can thus vary and impact differently the $\mathrm{Q}$ factors, explaining the narrowing of the large confidence interval with increasing radius. In a nutshell, the sidewall roughness observed in our sample is supposed to give a contribution $Q_{R}$ from $10^{4}$ to $10^{5}$. We attribute this roughness to the technological process but we cannot exclude any correlation with the presence of APBs even if typical dimensions do not fit.

The $Q_{R}$ contribution is not enough to explain the drop of $\bar{Q}$ down to 8000 as observed in our sample. A third contribution is needed. This can most likely come from absorption induced by APBs in the 
volume of the GaP layer. It has indeed been shown theoretically that APBs act as deep centers, which result in a modification of the absorption properties especially within the band gap of GaP. From the work of Tea et al. we can expect a redshift of the band gap edge of $0.7 \mathrm{eV}$ so that absorption at $1.55 \mu \mathrm{m}$ is still affected by the exponential tail of the electronic contribution to the dielectric constant. From their work, the induced attenuation factor can be estimated to $\alpha \sim 1 \mathrm{~cm}^{-1}$. The effect of different values of $\alpha$ on the $Q_{i}$ is presented in Fig.3 d) with $Q_{A P D}=m / \alpha R$. An attenuation factor $\alpha \sim 2.5 \pm 0.2 \mathrm{~cm}^{-1}$ allows to obtain a $\mathrm{Q}$ factor equal to $\bar{Q}$ at $S_{c, l o n g}$. This attenuation corresponds to linear losses of $10 \pm 1 \mathrm{~dB} / \mathrm{cm}$, for a density of APBs of $8000 \mathrm{~mm}^{-1}$. The APB-limited Q factor would then be of 45000 . The estimated value of $10 \mathrm{~dB} / \mathrm{cm}$ is in the same range as typical losses recently reported in $\mathrm{GaP}$ waveguides grown on III-V substrates, and is thus compatible with the realization of practical individual nonlinear devices. [27]

To conclude, we demonstrated GaP microdisks monolithically integrated on silicon. These photonic resonators show a unique random distribution of the III-V crystal polarity that could be used to engineer the nonlinear properties of the material. The material optical losses measured in the microdisks of $\sim 10 \mathrm{~dB} / \mathrm{cm}$ are compatible with the realization of practical nonlinear components at lowest costs thanks to $\mathrm{Si}$ substrates and the minimal III-V material deposition. The development of larger photonic circuits based on this random polarity platform now requires the transfer of the GaP membrane onto dielectric substrates and refinement of technological processing to reach APB-limited optical losses.

Funding. French National Research Agency (ANR-17-CE24-0019) and Région Bretagne.

Acknowledgment. The authors acknowledge the technological platform NanoRennes, member of Renatech network in which the samples were grown and processed.

Disclosures. The authors declare no conflicts of interest.

\section{References}

1. M. L. Davenport, S. Skendžić, N. Volet, J. C. Hulme, M. J. R. Heck, and J. E. Bowers, IEEE Journal of Selected Topics in Quantum Electronics 22, 78 (2016).

2. D. T. Spencer, T. Drake, T. C. Briles, J. Stone, L. C. Sinclair, C. Fredrick, Q. Li, D. Westly, B. R. Ilic, A. Bluestone, N. Volet, T. Komljenovic, L. Chang, S. H. Lee, D. Y. Oh, M.-G. Suh, K. Y. Yang, M. H. P. Pfeiffer, T. J. Kippenberg, E. Norberg, L. Theogarajan, K. Vahala, N. R. Newbury, K. Srinivasan, J. E. Bowers, S. A. Diddams, and S. B. Papp, Nature 557, 81 (2018).

3. A. Martin, D. Sanchez, S. Combrié, A. de Rossi, and F. Raineri, Opt. Lett., OL 42, 599 (2017).

4. D. J. Wilson, K. Schneider, S. Hönl, M. Anderson, Y. Baumgartner, L. Czornomaz, T. J. Kippenberg, and P. Seidler, Nat. Photonics 14, 57 (2020).

5. A. Bernard, J.-M. Gérard, G. Leo, and I. Favero, in Nonlinear Optics - Novel Results in Theory and Applications (2018).

6. H. Jin, F. M. Liu, P. Xu, J. L. Xia, M. L. Zhong, Y. Yuan, J. W. Zhou, Y. X. Gong, W. Wang, and S. N. Zhu, Phys. Rev. Lett. 113, 103601 (2014).

7. J. Lu, J. B. Surya, X. Liu, A. W. Bruch, Z. Gong, Y. Xu, and H. X. Tang, Optica, OPTICA 6, 1455 (2019).

8. C. Cornet, Y. Léger, and C. Robert, (ISTE-Elsevier, 2016).

9. S. Chen, W. Li, J. Wu, Q. Jiang, M. Tang, S. Shutts, S. N. Elliott, A. Sobiesierski, A. J. Seeds, I. Ross, P. M. Smowton, and H. Liu, Nature Photonics 10, 307 (2016).
10. P. G. Schunemann, L. Mohnkern, A. Vera, X. S. Yang, A. C. Lin, J. S. Harris, V. Tassev, and M. Snure, in (OSA, 2012), p. ITh5B.5.

11. I. Lucci, S. Charbonnier, L. Pedesseau, M. Vallet, L. Cerutti, J.-B. Rodriguez, E. Tournié, R. Bernard, A. Létoublon, N. Bertru, A. Le Corre, S. Rennesson, F. Semond, G. Patriarche, L. Largeau, P. Turban, A. Ponchet, and C. Cornet, Phys. Rev. Materials 2, 060401 (2018).

12. K. Volz, A. Beyer, W. Witte, J. Ohlmann, I. Németh, B. Kunert, and W. Stolz, J. Cryst. Growth 315, 37 (2011).

13. A. C. Lin, M. M. Fejer, and J. S. Harris, Journal of Crystal Growth 363, 258 (2013).

14. M. Baudrier-Raybaut, R. Haïdar, Ph. Kupecek, Ph. Lemasson, and E. Rosencher, Nature 432, 374 (2004).

15. P. Guillemé, Y. Dumeige, J. Stodolna, M. Vallet, T. Rohel, A. Létoublon, C. Cornet, A. Ponchet, O. Durand, and Y. Léger, Semicond. Sci. Technol. 32, 065004 (2017).

16. E. Tea, J. Vidal, L. Pedesseau, C. Cornet, J.-M. Jancu, J. Even, S. Laribi, J.-F. Guillemoles, and O. Durand, Journal of Applied Physics 115, 063502 (2014).

17. Y. P. Wang, J. Stodolna, M. Bahri, J. Kuyyalil, T. N. Thanh, S. Almosni, R. Bernard, R. Tremblay, M. D. Silva, A. Létoublon, T. Rohel, K. Tavernier, L. Largeau, G. Patriarche, A. L. Corre, A. Ponchet, C. Magen, C. Cornet, and O. Durand, Applied Physics Letters 107, 191603 (2015).

18. P. Guillemé, M. Vallet, J. Stodolna, A. Ponchet, C. Cornet, A. Létoublon, P. Féron, O. Durand, Y. Léger, and Y. Dumeige, Optics Express 24, 14608 (2016).

19. C. Cornet, S. Charbonnier, I. Lucci, L. Chen, A. Létoublon, A. Alvarez, K. Tavernier, T. Rohel, R. Bernard, J.-B. Rodriguez, L. Cerutti, E. Tournié, Y. Léger, M. Bahri, G. Patriarche, L. Largeau, A. Ponchet, P. Turban, and N. Bertru, Phys. Rev. Materials 4, 053401 (2020).

20. W. Guo, A. Bondi, C. Cornet, A. Létoublon, O. Durand, T. Rohel, S. Boyer-Richard, N. Bertru, S. Loualiche, J. Even, and A. Le Corre, Applied Surface Science 258, 2808 (2012).

21. P. Guillemé, J. Stervinou, T. Rohel, C. Cornet, D. Gachet, S. Balac, F. Mahé, Y. Dumeige, and Y. Léger, Opt. Lett., OL 43, 1766 (2018).

22. C. P. Michael, M. Borselli, T. J. Johnson, C. Chrystal, and O. Painter, Optics Express 15, 4745 (2007).

23. Q. Li, A. A. Eftekhar, Z. Xia, and A. Adibi, Phys. Rev. A 88, 033816 (2013).

24. M. Borselli, T. J. Johnson, and O. Painter, Opt. Express, OE 13, 1515 (2005).

25. D. Parrain, C. Baker, G. Wang, B. Guha, E. G. Santos, A. Lemaitre, P. Senellart, G. Leo, S. Ducci, and I. Favero, Optics Express 23, 19656 (2015).

26. J. Heebner, R. Grover, T. Ibrahim, and Heebner-Grover-Ibrahim, Springer Series in Optical Sciences No. 138 (Springer, 2008).

27. A. P. Anthur, Z. Haizhong, Y. Akimov, O. Junrong, D. Kalashnikov, A. I. Kuznetsov, and L. Krivitsky, arXiv:2001.06142 [physics] (2020). 